\title{
Over Recovery in Low Pressure Sparkgaps
}

\author{
K. V. Nagesh, P. H. Ron, G. R. Nagabhushana, and R. S. Nema
}

\begin{abstract}
The recovery characteristics of the low pressure sparkgaps in the time interval of $300 \mu \mathrm{s}$ to $50 \mathrm{~ms}$ by the influence of 1) gap spacings of 2.5 and $10 \mathrm{~mm}, 2$ ) pulse voltages of 45 $\mathrm{kV}$ rising in $2.5 \mu \mathrm{s}$ for positive and negative polarities, and 3) the pressure range of $1.3-34.7 \mathrm{~Pa}$, have been determined for hydrogen, argon, and deuterium gases. A two-pulse system, capable of generating two pulses rising to $45 \mathrm{kV}$ each in 2.5 $\mu \mathrm{s}$ and separated by a variable delay of $100 \mu \mathrm{s}$ to $50 \mathrm{~ms}$, has been used to study the recovery characteristics. It has been observed that the breakdown voltage under the second pulse is higher than the breakdown voltage under the first pulse along the left-hand side of Paschen's' characteristics [1] and this has been defined as over recovery $(>100 \%$ recovery) in these studies. The over recovery was found to be up to $120 \%$ and is dependent on the gas pressure $\{<23 \mathrm{~Pa}$ for argon, $<12.1$ $\mathrm{Pa}$ for $\mathrm{H}_{2},<4.5 \mathrm{~Pa}$, - ve polarity, and $<14.3 \mathrm{~Pa}$, + ve polarity for $\mathrm{D}_{2}$ gases $\}$, discharge current magnitude $(>1 \mathrm{kA})$, and its reversal. This over recovery in low pressure sparkgaps is due to pressure reduction in the gap after the first pulse discharge. This finding seems to have been reported for the first time. The recovery times are independent of gas pressure for negative polarity. It increases with pressure for positive polarity. It increases with increase in molecular weight of the gas in the gap. Shorter gaps recover faster than longer gaps. The experimental setups, principal of over recovery, measurement of breakdown voltages, and currents, results and discussions are presented here.
\end{abstract}

Index Terms - Gas sparkgaps, low pressure sparkgaps, over recovery, recovery times, sparkgaps, sparkgap recovery.

\section{INTRODUCTION}

$\mathbf{T}$ WE recovery of the sparkgap between the first and subsequent pulses is a very important phenomenon in repetitive pulsed sparkgaps. Generally the sparkgaps recovering to its first pulse breakdown voltage conditions (100\%) have been reported [2]-[6]. The low pressure sparkgaps recover to greater than its breakdown voltages for second pulse (SPBDV) compared to first pulse (FPBDV) under certain conditions. This is defined as over recovery in these studies. The first pulse discharge produces plasma in the sparkgap. This momentarily increases the gap pressure and temperature to $\sim 1.6 \times 10^{5}$ $\mathrm{Pa}$ [2] and $\sim 5000 \mathrm{~K} \mathrm{[3],} \mathrm{[4]} \mathrm{in} \mathrm{the} \mathrm{discharge} \mathrm{column.} \mathrm{The}$ plasma expands and diffuses in all directions. The pressure

Manuscript received April 28, 1998; revised November 12, 1998.

K. V. Nagesh and P. H. Ron are with the Accelerator and Pulse Power Division, Bhabha Atomic Research Centre, Trombay, Mumbai, 400085, India (e-mail: epps@apsara.barc.ernet.in).

G. R. Nagabhushana and R. S. Nema are with the Department of High Voltage Engineering, Indian Institute of Science, Malleswaram, Bangalore, 560012, India.

Publisher Item Identifier S 0093-3813(99)02286-9. reduces below the normal operating pressure inversely to the temperature of the plasma. If the plasma expansion supported by vacuum pumping dominates the gas inlet, the pressure in the gap volume reduces below normal and it results in over recovery of the gap. It is assumed that all ionizations in the gap due to first pulse discharge are diffused to the walls by this time. If the temperature and pressure have been reduced to normal by the time ionizations are diffused, the gap recovers to full recovery. It results in under recovery for delay times lower than the above, wherein the pressure in the gap is higher than the normal or the left-over ionizations in the gap leads to breakdown at lower voltages. The effect of gap spacing, gas species, current magnitude and its reversal, and gap pressure on the recovery time of the low pressure sparkgaps have been studied and reported here.

\section{EXPERIMENTAL SETUP}

The experimental setup is shown in Fig. 1. It consists of a low pressure sparkgap connected to a vacuum system, fitted with a $5 \times 10^{-6} \mathrm{~m}^{3} / \mathrm{s}$ rotary pump, $1 \times 10^{-3} \mathrm{~m}^{3} / \mathrm{s}$ oil diffusion pump (silicone oil), and a liquid nitrogen trap. The stainless steel electrodes assembled at the centre of polymethyl methacrylate (PMMA) chamber is mounted inside the stainless steel chamber. A PMMA tube is fixed on this flange enclosing the sparkgap for preventing long path breakdowns to the chamber. The electrodes are of Rogowskii profile, machined on a CNC lathe, for a uniform electric field between electrodes.

High purity Iolar-S argon (999 965 ppm) and Iolar-2 hydrogen $(999888.9 \mathrm{ppm})$ gases are used in these experiments. The deuterium gas (99.7\%) used in these experiments is produced by electrolysis of heavy water, using palladium filter. The gas is let in through the central hole of the electrode E2. This results in maximum gas pressure in the gap volume between the flat portion of the electrodes [5] and it reduces toward walls. As a consequence of this, the breakdowns occur over the central flat area of the electrodes for pressures to the lefthand side of Paschen's minimum. The gas inlet hole introduces slight nonuniformity to the present sparkgap configuration. This results in higher breakdown voltages under positive polarity compared to negative polarity [6]. The experimental chamber evacuated to $1.3 \times 10^{-3} \mathrm{~Pa}$, is filled with the required gas up to $\sim 133 \mathrm{~Pa}$ pressure and evacuated again to $1.3 \times 10^{-3} \mathrm{~Pa}$, before starting the experiments. This flushing process is repeated four times to maintain the purity of gas.

A two-pulse system consisting of two high voltage power supplies based on Tesla transformers charging co-axial cable 


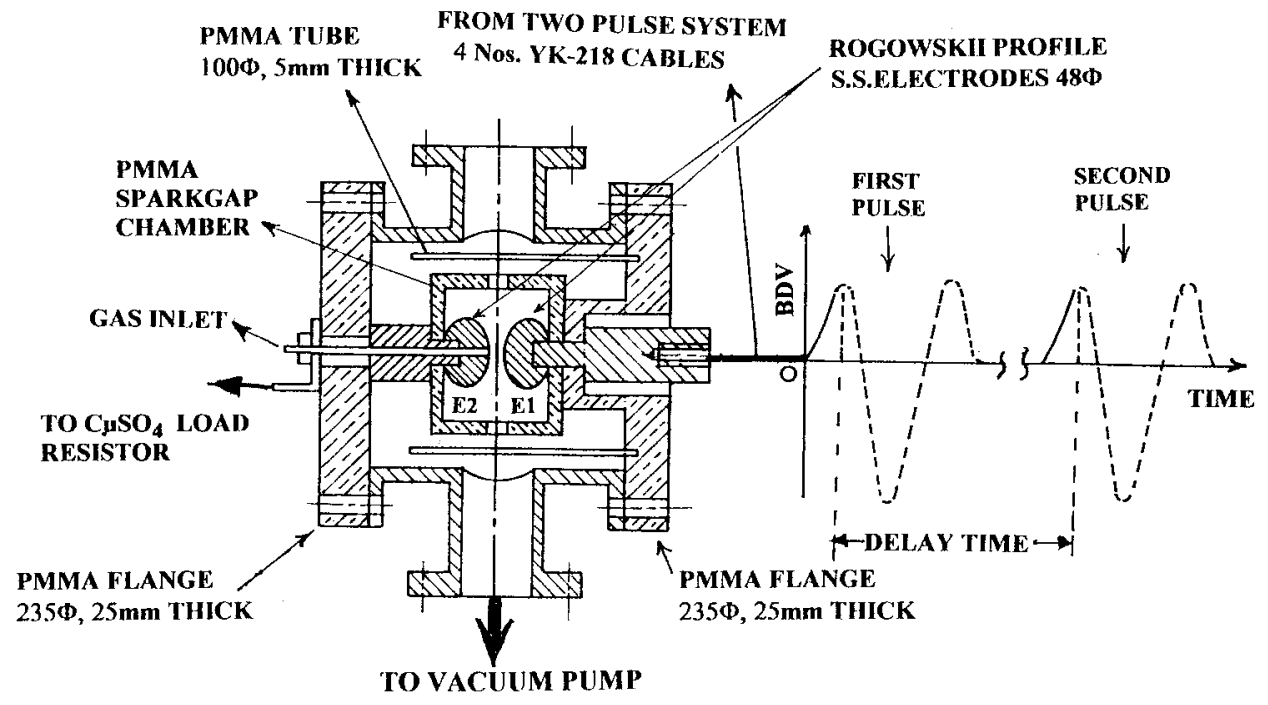

Fig. 1. Experimental chamber of low pressure sparkgap.

(a)

(b)

(c)

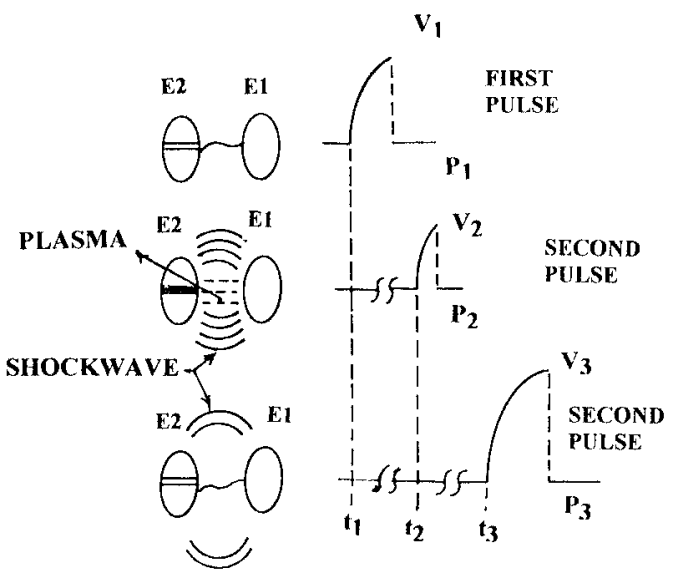

Fig. 2. Sequence of recovery process in low pressure sparkgaps.

transmission lines, having individual control for the two trigger pulses from silicon controlled rectifiers (SCR's) trigger pulse generators and the low pressure sparkgap has been used in these studies. Four YK-218 cables of $8 \mathrm{~m}$ length connected to the electrode E1 of low pressure sparkgap through PMMA insulator and a copper tube for co-axial return path, are used as transmission lines. The two YK-218 cables are connected to two Tesla transformers at the other ends. The first voltage pulse having a rise time of $\sim 2.5 \mu \mathrm{s}$, charges the co-axial cable transmission line to a peak voltage. Depending upon the pressure inside the gap volume, gap spacing and the applied voltage, the sparkgap breaks down and discharges the energy stored in the transmission line to the copper sulphate load. The second voltage pulse having a rise time of $\sim 2.5 \mu \mathrm{s}$, applied after a pre-set delay varying from $300 \mu \mathrm{s}$ to $50 \mathrm{~ms}$, charges the co-axial cable transmission line to a peak value. Depending upon the recovery condition of the sparkgap, it breaks down and discharges the energy stored in the transmission line to the copper sulphate load.

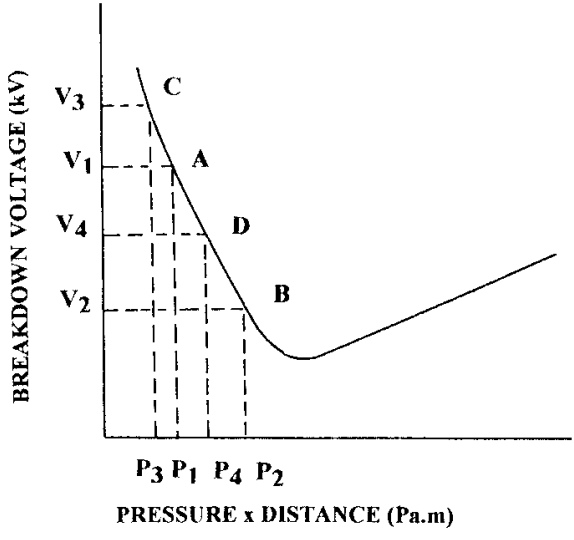

Fig. 3. Pulsed Paschen's characteristics.

\section{Principal of Over Recovery}

Fig. 2 shows the sequence of operation in a low pressure sparkgap. Fig. 3 shows the general Paschen's characteristics. The first pulse is applied to the electrode E1 at a time $t_{1}$ with a pressure in the gap $P_{1}$ as shown in Fig. 2(a). The gap breaks down at a voltage $V_{1}$ (point $\mathrm{A}$ ) and produces plasma and shockwaves in the gap as shown in Fig. 2(b). The possible ways the breakdowns of second and subsequent pulses are affected can be explained as follows. The first pulse breakdown increases temperature and pressure in the discharge column of the gap. The temperature and pressure in the gap starts reducing due to diffusion of plasma to the walls, resulting in recovery of the gap. The pressure in the gap reduces to $P_{2}$ at a time $t_{2}$ (point $\mathrm{B}$ ). If the second pulse is applied at this instant, the gap breaks down at a voltage $V_{2}<V_{1}$ as shown in Fig. 3 (point B). This shows that the gap has not recovered to its pre-breakdown status (under recovery). It can be seen from Fig. 3, that increase in pressure results in reduction of the breakdown voltage and vice-versa. If the second pulse is applied at a later instant $t_{3}$, at which time if the pressure 


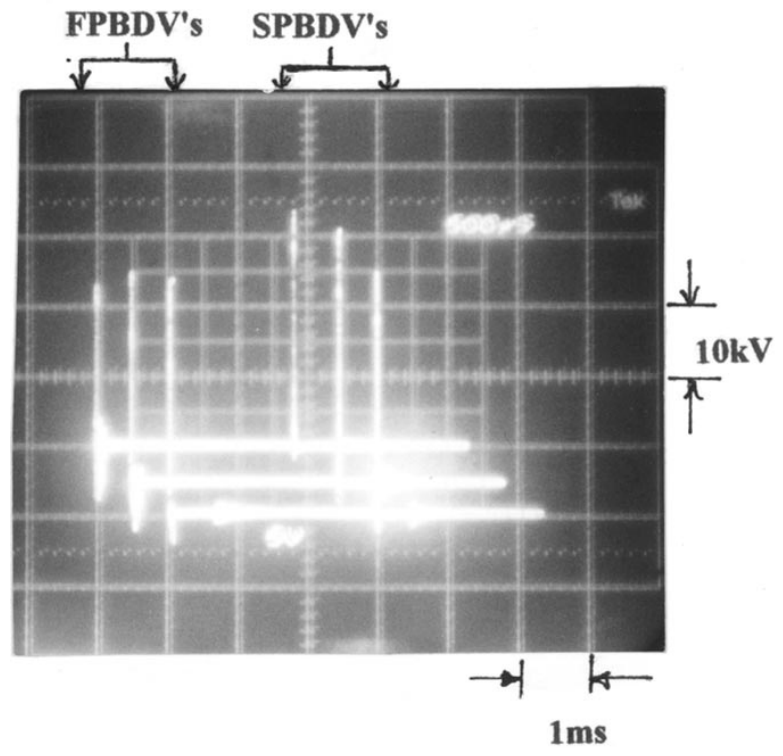

(a)

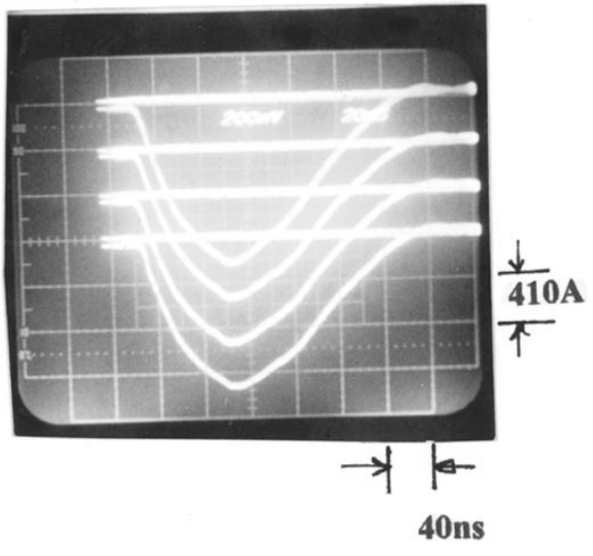

(b)

Fig. 4. First and second voltage and current pulse waveforms. Argon gas, $3.2 \mathrm{~Pa}, 10 \mathrm{~mm}$ gap, and $3.3 \Omega$ load. (a) Voltage pulses, +ve polarity and (b) current pulses, -ve polarity.

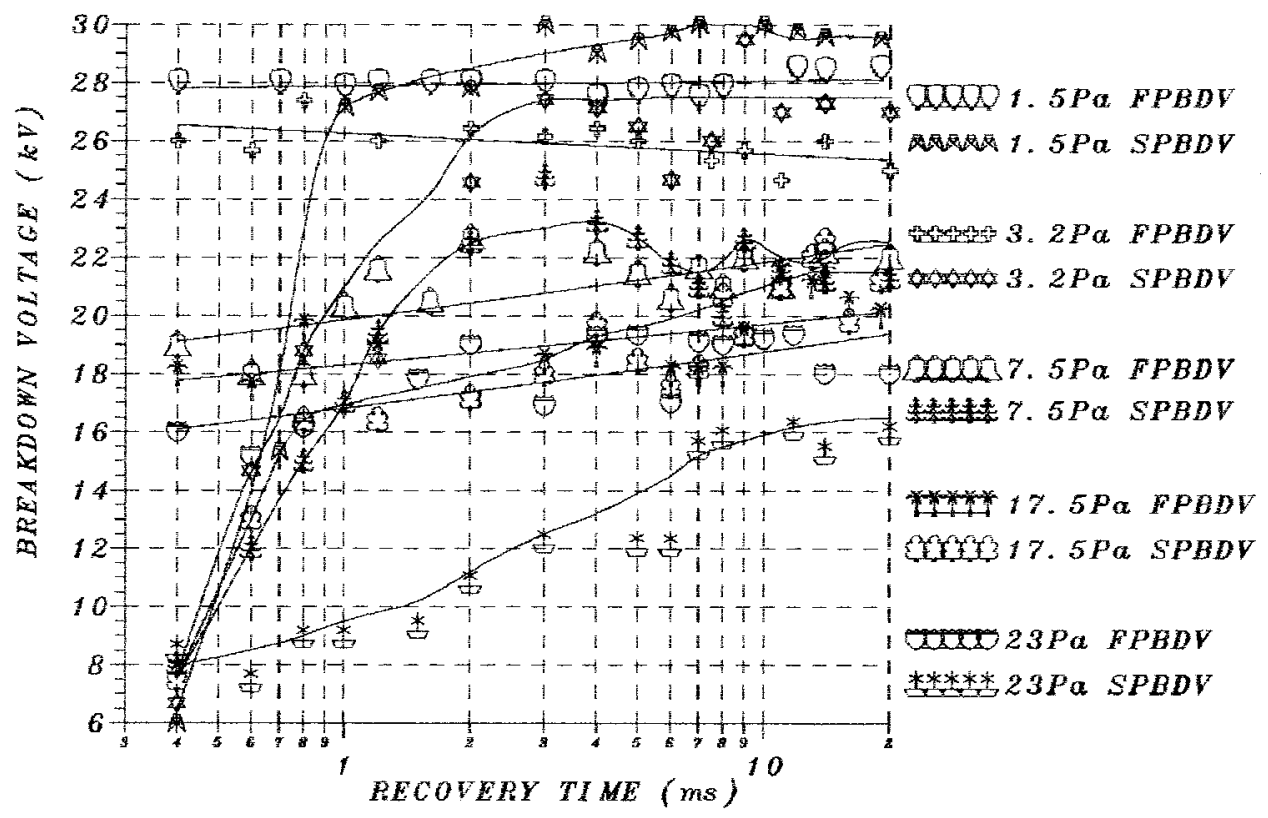

Fig. 5. Breakdown voltage as a function of recovery time characteristics, argon gas, $10 \mathrm{~mm}$ gap, -ve polarity, no current reversal, and $5.2 \Omega$ load.

$P_{3}$ is equal to $P_{1}$, it will breakdown at a voltage $V_{3}$. It is called full recovery if $V_{3}$ is equal to $V_{1}$. It is presumed that all ionizations in the gap have been neutralized by this time. Alternatively if the pressure $P_{3}$ is less than $P_{1}$ (point C), it will breakdown at a voltage $V_{3}>V_{1}$ as shown in Fig. 2(c) and Fig. 3, which is called over recovery. This over recovery is possible only in case of low pressure sparkgaps where the pressure can reduce below nominal operating pressure under large discharge currents. The interval from $\mathrm{A}$ to $\mathrm{B}$ and to $\mathrm{C}$ is called over-recovery time and the corresponding breakdown voltages are over-recovery voltages.

\section{Measurement of Gap Spacing, Gap PRESSURe, ReCovery Voltages, AND CURRENTS}

The sparkgap electrodes are assembled in PMMA chambers as shown in Fig. 1. The sparkgap spacing has been set to an accuracy of $98 \%$ for $2.5 \mathrm{~mm}$ gap and $99.5 \%$ for $10 \mathrm{~mm}$ gap. The gap is mounted inside the stainless steel chamber connected to a vacuum system. The electrodes are conditioned by glow discharge at $100 \mu \mathrm{A}$ and $2 \mathrm{~Pa}$ gap pressure for $15 \mathrm{~min}$. This removes the surface impurities and micro projections, which are not removed by polishing 


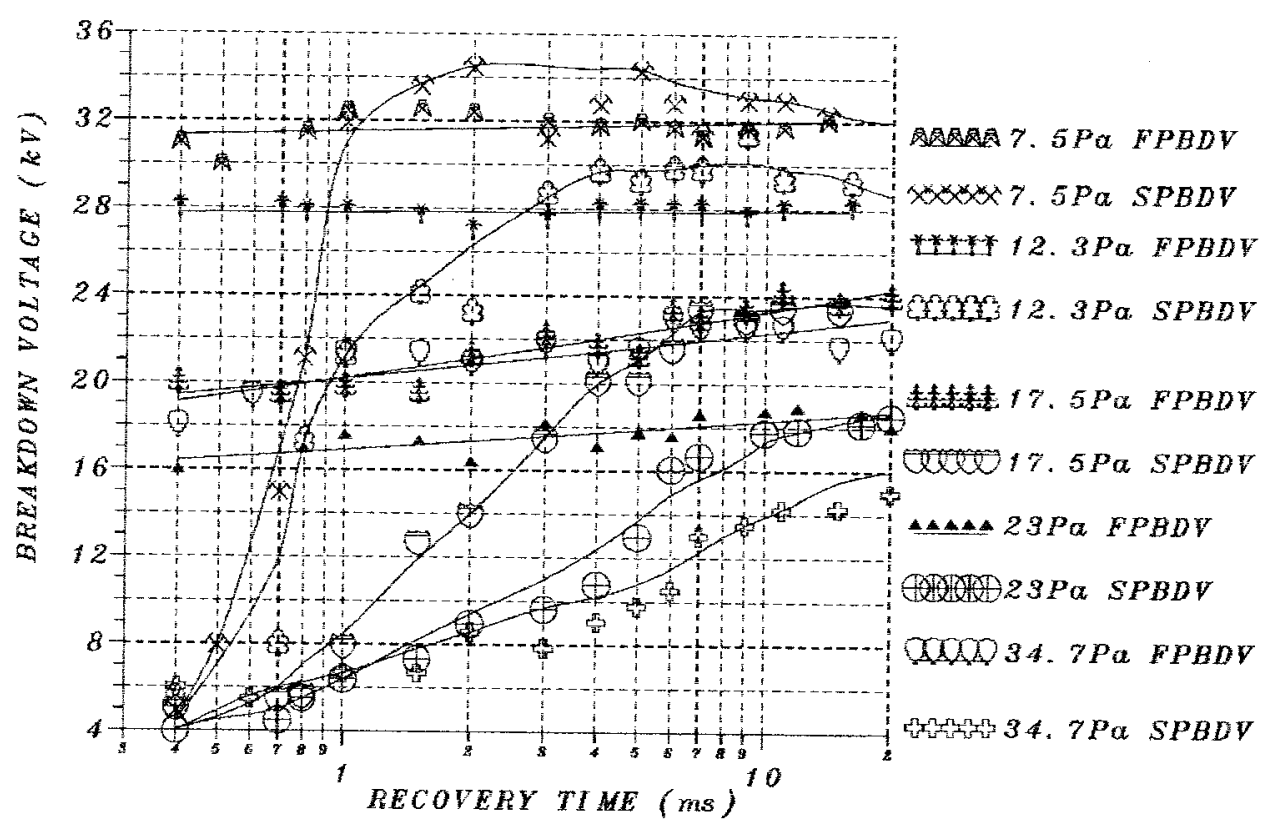

Fig. 6. Breakdown voltage as a function of recovery time characteristics, argon gas, $10 \mathrm{~mm}$ gap, +ve polarity, no current reversal, and $5.2 \Omega$ load.

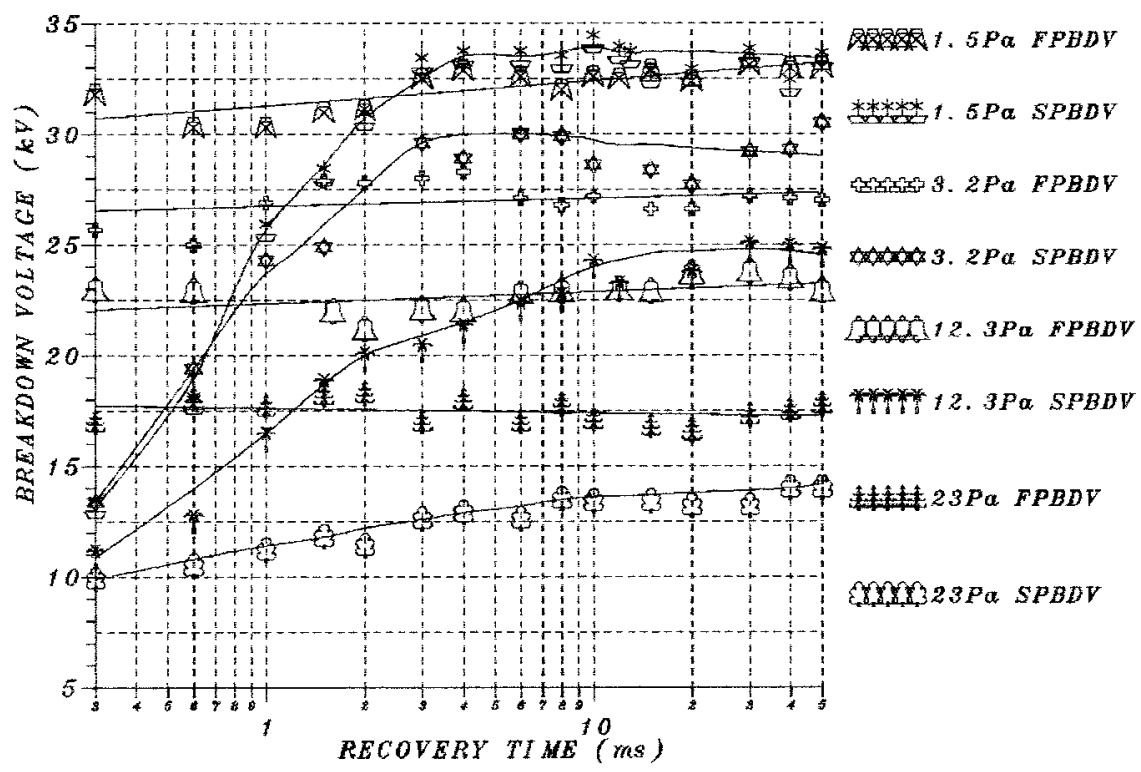

Fig. 7. Breakdown voltage as a function of recovery time characteristics, argon gas, $10 \mathrm{~mm}$ gap, - ve polarity, $50 \%$ current reversal, and $3.3 \Omega$ load.

and cleaning. In addition to this, the electrodes are conditioned between trials of two gap pressures under recovery experiments similar to initial conditioning of electrodes. This removes the micro projections developed during previous experiments.

It is not possible to measure the pressure inside the gap volume during the sparkgap recovery experiments, due to discharge to the convectron pressure gauge. Due to this problem, the pressure inside the sparkgap at the time of recovery studies is measured, by simulating identical conditions, using convectron pressure gauges having an accuracy of one digit $(1 \mathrm{mtorr}=0.133 \mathrm{~Pa}$ ). The measured gap pressure has a lower accuracy of $10-30 \%$ at $1.0 \mathrm{~Pa}$ to $0.8-1.6 \%$ at $34.7 \mathrm{~Pa}$ for different gases.

The recovery voltages are measured using a $40 \mathrm{kV}, 6 \mathrm{~ns}$ rise time, $75 \mathrm{MHz}$, HV probe model P6015A along with $100 \mathrm{MHz}$ bandwidth, 7633 type analog storage oscilloscope supplied by M/S Tektronix Inc., USA. Only first pulse current has been recorded in these experiments using current viewing resistor $(0.009978 \Omega)$. Fig. 4(a)-(b) presents the oscillographically recorded sets of first and second pulse breakdown voltages and a set of first pulse currents for argon gas. The voltage waveforms show over recovery of the low pressure sparkgap. The overall error in measurement of pulsed BDV varies 


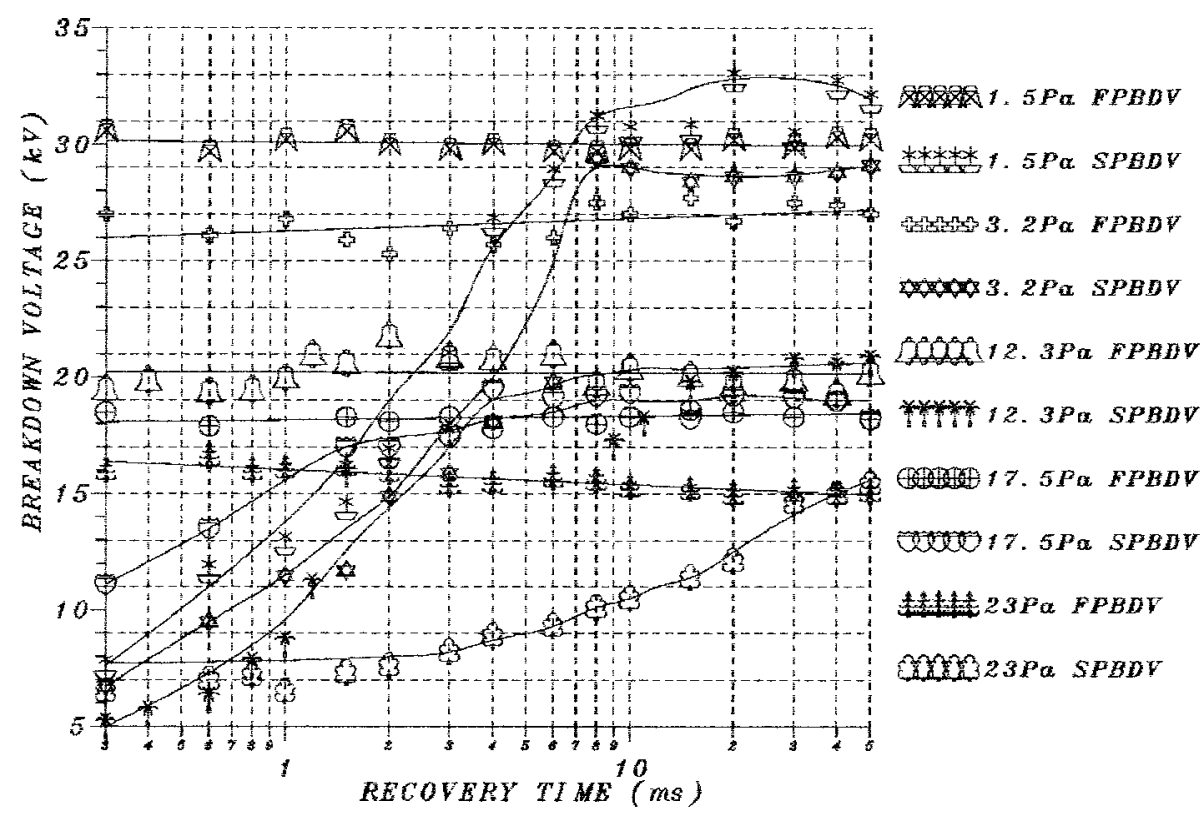

Fig. 8. Breakdown voltage as a function of recovery time characteristics, argon gas, $10 \mathrm{~mm}$ gap, +ve polarity, $50 \%$ current reversal, and $3.3 \Omega$ load.

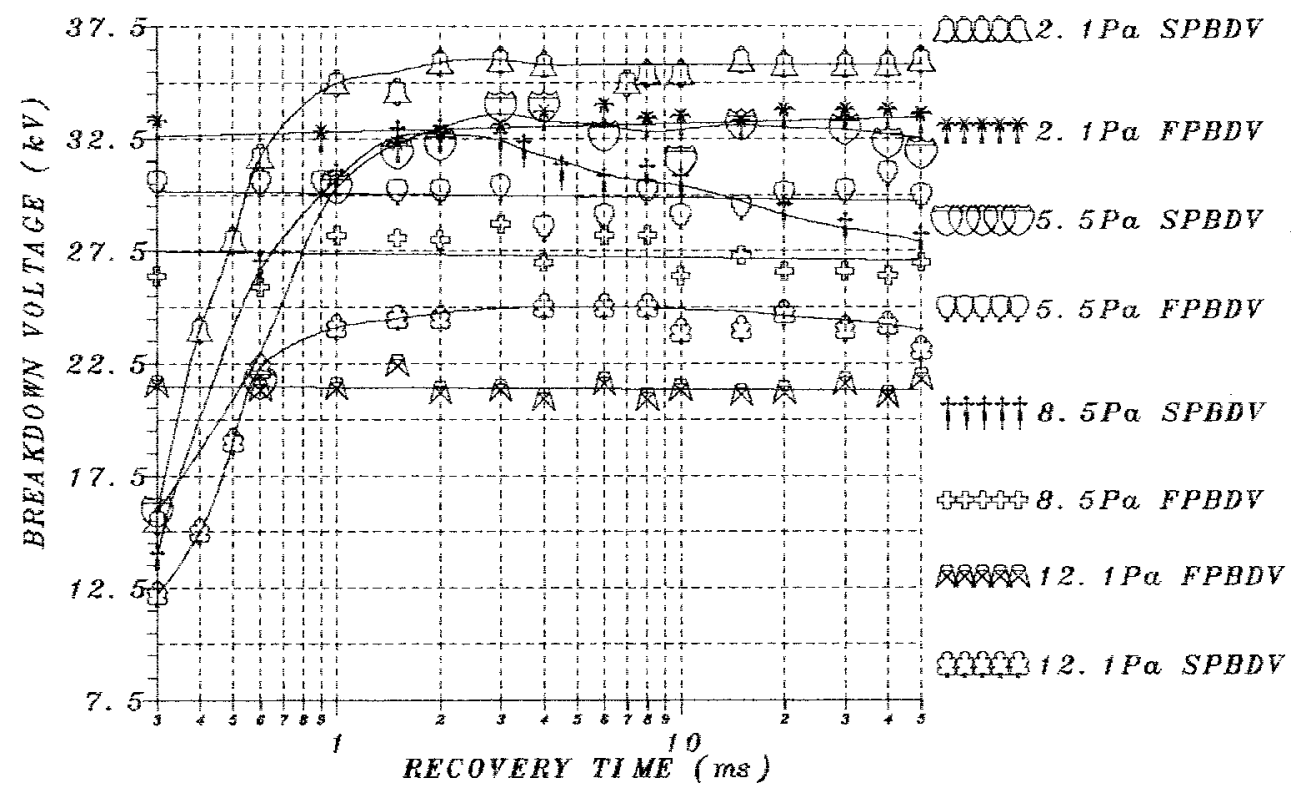

Fig. 9. Breakdown voltage as a function of recovery time characteristics, hydrogen gas, $10 \mathrm{~mm}$ gap, - ve polarity, $50 \%$ current reversal, and $3.3 \Omega$ load.

from $\sim 5 \%$ at low pressures to $\sim 2 \%$ at high pressures. The most probable breakdown voltages of recovery experiments are obtained using normal distribution [7], [8] out of ten breakdown voltages for first and second pulses. In Weibull two-parameter distribution [9]-[11], the lowest first pulse breakdown voltage (FPBDV) lies outside the $99 \%$ confidence interval of the shape parameter $\beta$. The second pulse breakdown voltages (SPBDV) with under recovery lie outside lower $99 \%$ confidence values and they lie outside upper $99 \%$ confidence interval for full recovery and over recovery. The breakdown voltages of first pulse follow Weibull three-parameter distribution and breakdown voltages of second pulse follow Weibull distribution of higher order [9]. Since it is appropriate to compare breakdown voltages under same distribution, normal distribution has been used in these studies.

\section{Recovery Times of Low Pressure Sparkgaps}

Figs. 5-14 present the breakdown voltage (BDV) as a function of recovery time characteristics. The pulsed BDV as a function of gap pressure characteristics are presented in Fig. 15 for both $-v e$ and +ve polarities. The full recovery time as a function of gap pressure characteristics are presented in Figs. 16 and 17. The first pulse BDV is consistent and lying within $\pm 5 \%$. The positive polarity BDV is higher than negative 


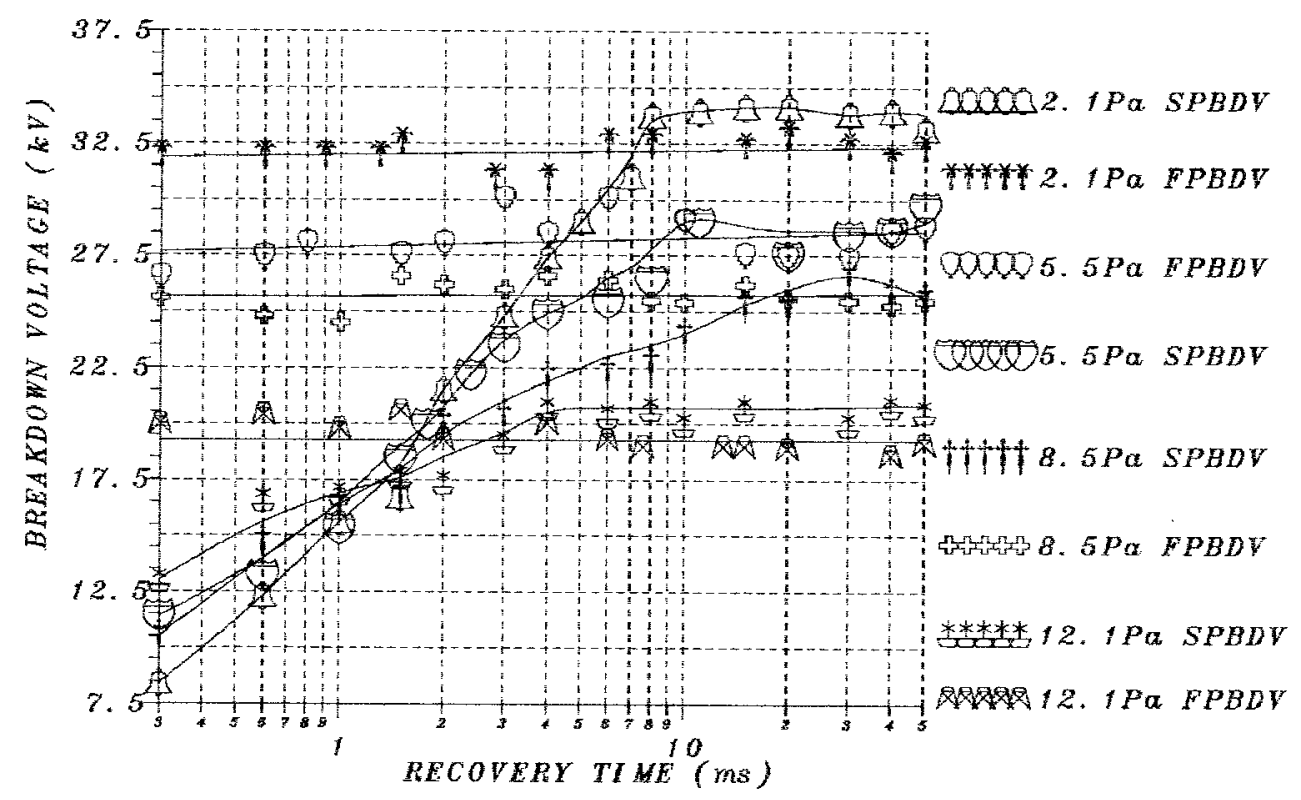

Fig. 10. Breakdown voltage as a function of recovery time characteristics, hydrogen gas, $10 \mathrm{~mm}$ gap, +ve polarity, $50 \%$ current reversal, and $3.3 \Omega$ load.

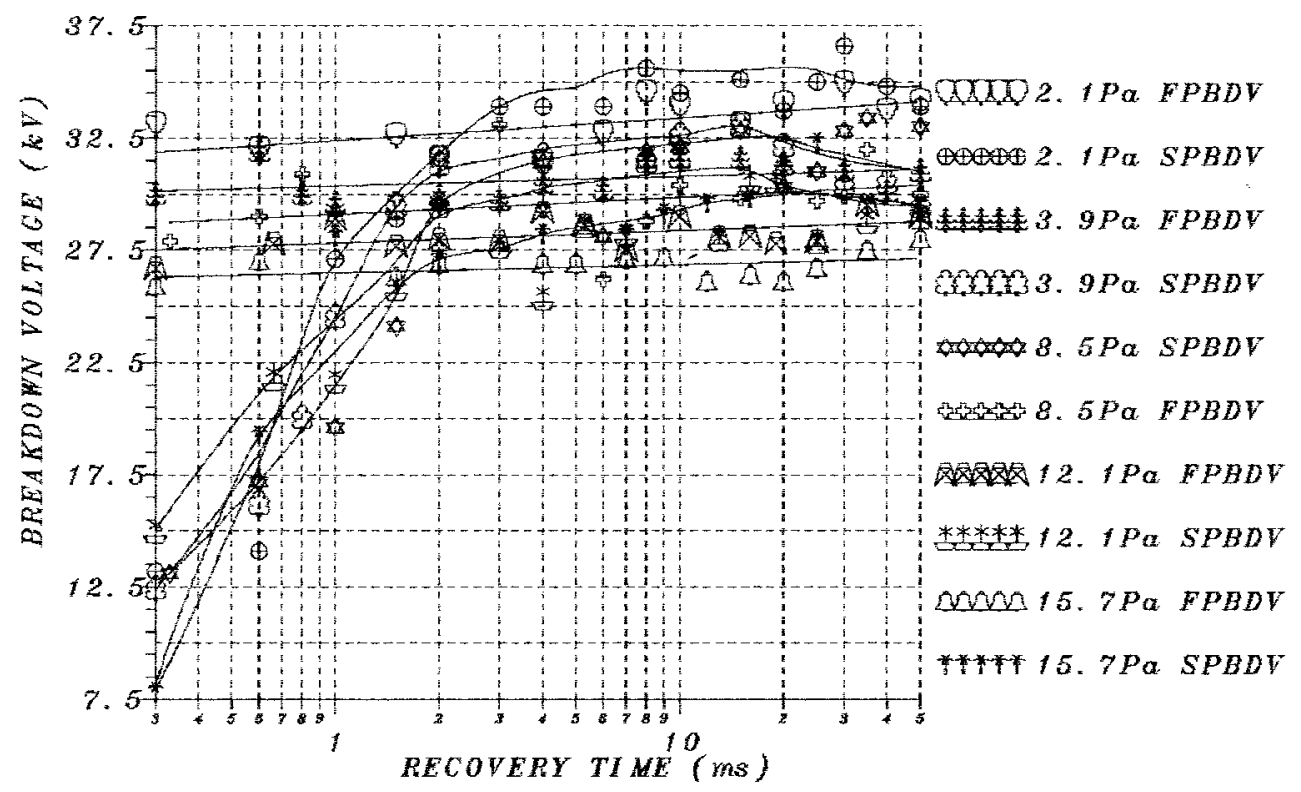

Fig. 11. Breakdown voltage as a function of recovery time characteristics, hydrogen gas, $2.5 \mathrm{~mm}$ gap, -ve polarity, no current reversal, and $5.2 \Omega$ load.

polarity BDV for all the gases for the experimental pressure range of recovery experiments. The BDV is higher in case of hydrogen gas (2.5 mm gap) compared to argon gas (10 mm gap) and deuterium gas (2.5 $\mathrm{mm}$ gap), respectively. The BDV of short gap $(2.5 \mathrm{~mm})$ is not in proportion to BDV of long gap $(10 \mathrm{~mm})$ in case of hydrogen gas. This means that pd is not a good variable for comparing breakdowns at two different conditions under the present sparkgap configuration [6].

The over recovery of the gap has been observed up to pressures of 1) $<23 \mathrm{~Pa}$ for argon, 2) $\leq 12.1 \mathrm{~Pa}$, for hydrogen, $3) \leq 4.5 \mathrm{~Pa}$, -ve polarity and $\leq 14.3 \mathrm{~Pa}$, +ve polarity, for deuterium gases. The short gap recovers faster than long gaps in case of hydrogen gas. The over recovery is predominant in case of short gaps and large current reversals compared to long gaps and no current reversals. The probabilities of over recovery increases with increase in discharge current. The full recovery time is fairly consistent with increase in pressure for negative polarity. It increases with increase in pressure for positive polarity. It is nearly constant in case of deuterium gas, +ve polarity. The second pulse BDV oscillates over the first pulse BDV after full recovery (similar to under-damped oscillations in a RLC network). The frequency of oscillation decreases with reduction in pressure. The present results show that the frequency of oscillation is minimum for pressure of 


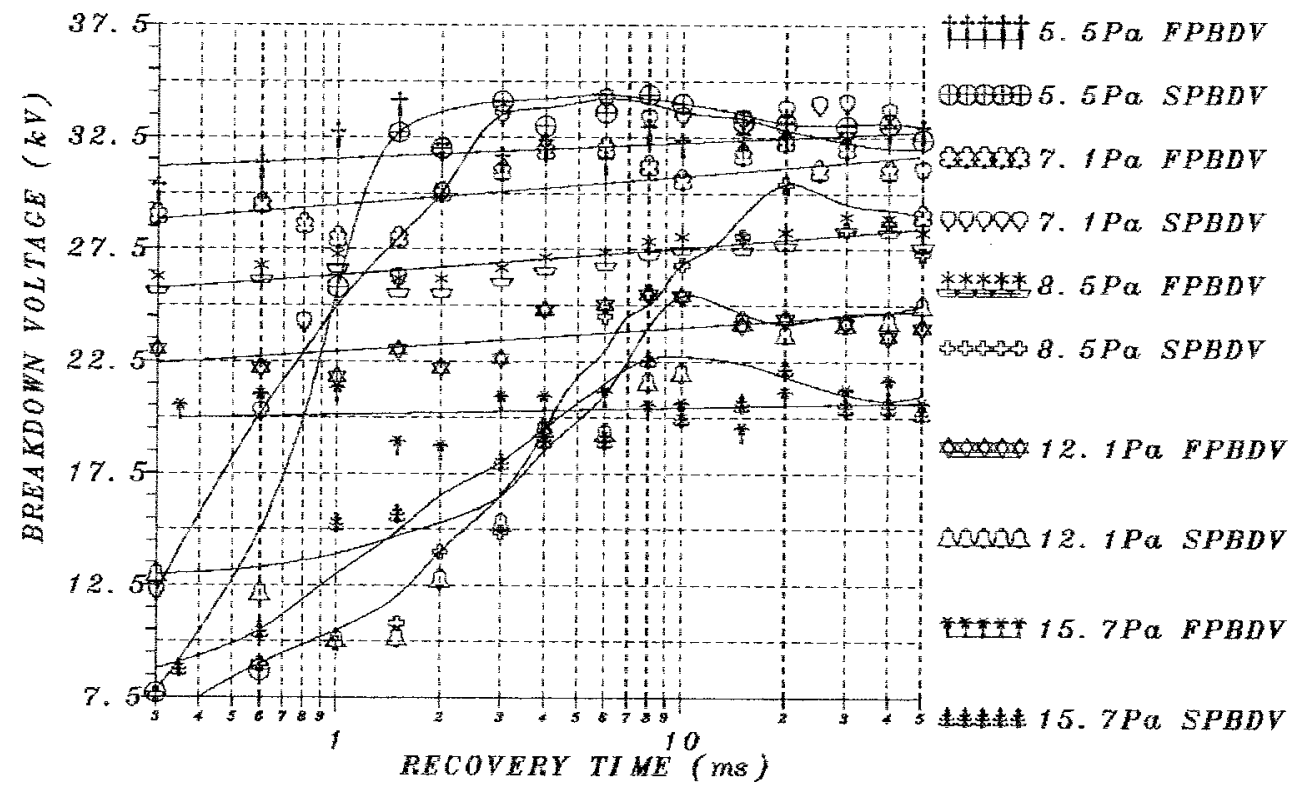

Fig. 12. Breakdown voltage as a function of recovery time characteristics, hydrogen gas, $2.5 \mathrm{~mm}$ gap, +ve polarity, no current reversal, and $5.2 \Omega$ load.

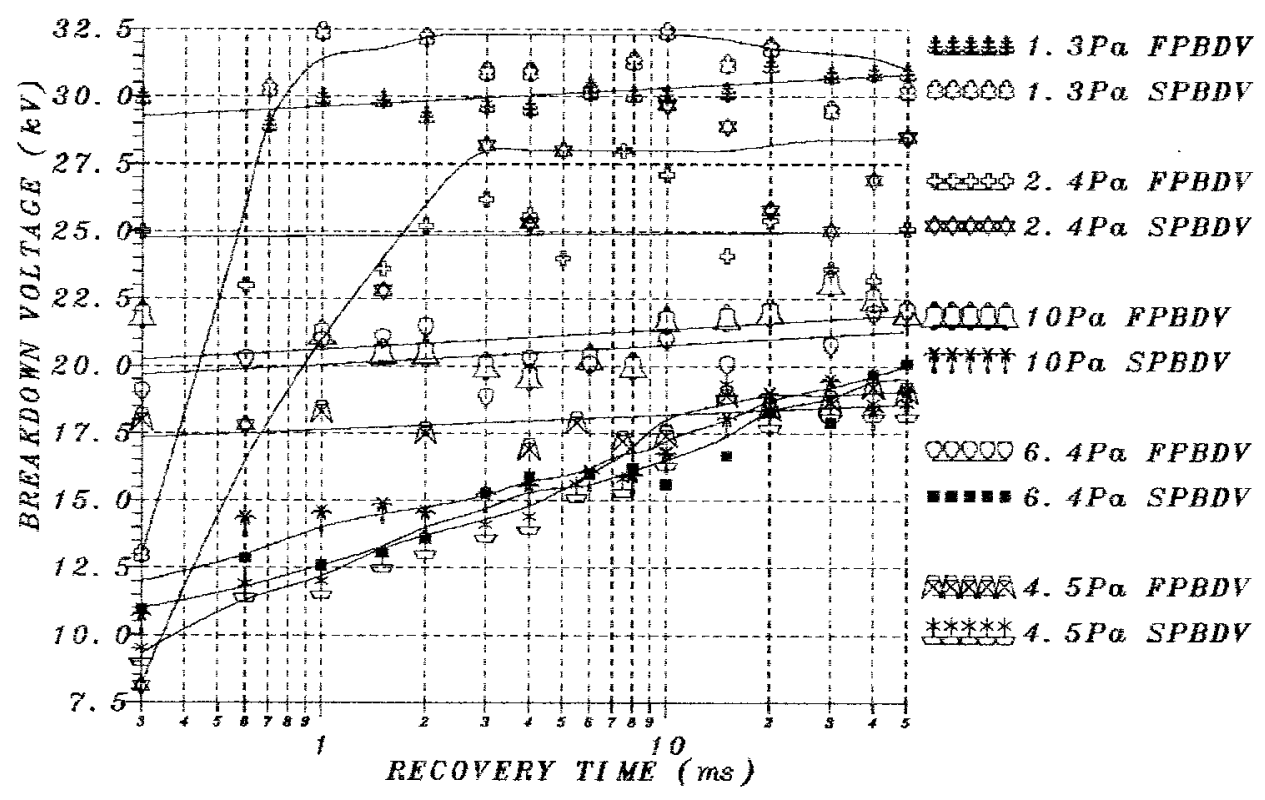

Fig. 13. Breakdown voltage as a function of recovery time characteristics, deuterium gas, $2.5 \mathrm{~mm}$ gap, - ve polarity, no current reversal, and $5.2 \Omega$ load.

1.5 $\mathrm{Pa}$ and maximum for $7.5 \mathrm{~Pa}$ for argon gas. This trend is similar for hydrogen and deuterium gases.

The over recovery is significant considering the spread in BDV's. The spread of BDV's are plotted for 2.1 and 12.1 Pa gap pressure of hydrogen gas, $3.3 \Omega$ with $50 \%$ current reversal in Fig. 18. The BDV's of the first and second pulses (ordered) at $2 \mathrm{~ms}$ delay time are $30,30,32,32,32,32,33$, $34,35,35 \mathrm{kV}$, and $33,33,34,34,36,36,36,38,38,40$ $\mathrm{kV}$, respectively. The average BDV's are 32.5 and $35.8 \mathrm{kV}$, respectively. The percentage of BDV greater than $32.5 \mathrm{kV}$ are 40 and $100 \%$ for first and second pulses, respectively. The percentage of BDV's greater than $35.8 \mathrm{kV}$ are nil and $60 \%$ for first and second pulses, respectively. The above characteristics clearly shows that the over recovery is significant considering the spread in breakdown voltages. This is applicable to argon and deuterium gases also.

The over recovery in low pressure sparkgaps is due to pressure reduction in the gap volume after the first pulse discharge, compared to nominal pressures. To estimate the change in pressures during discharge, $\mathrm{BDV}$ as a function of recovery time characteristics (Figs. 5-14) as well as pulsed BDV as a function of gap pressure characteristics (Fig. 15) are used (method similar to that of Tsuruta. [3], [4]). The second pulse BDV's are noted against delay times from recovery 


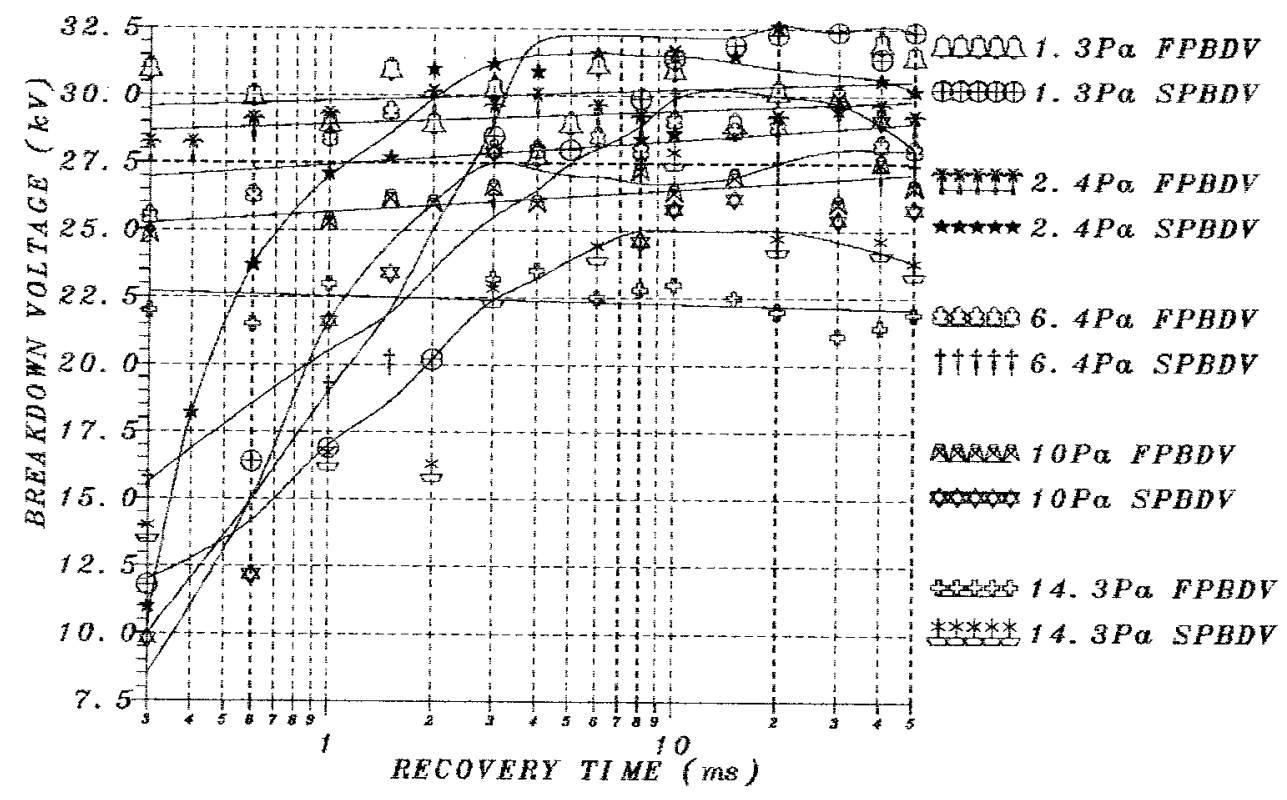

Fig. 14. Breakdown voltage as a function of recovery time characteristics, deuterium gas, $2.50 \mathrm{~mm}$ gap, +ve polarity, no current reversal, and $5.2 \Omega$ load.

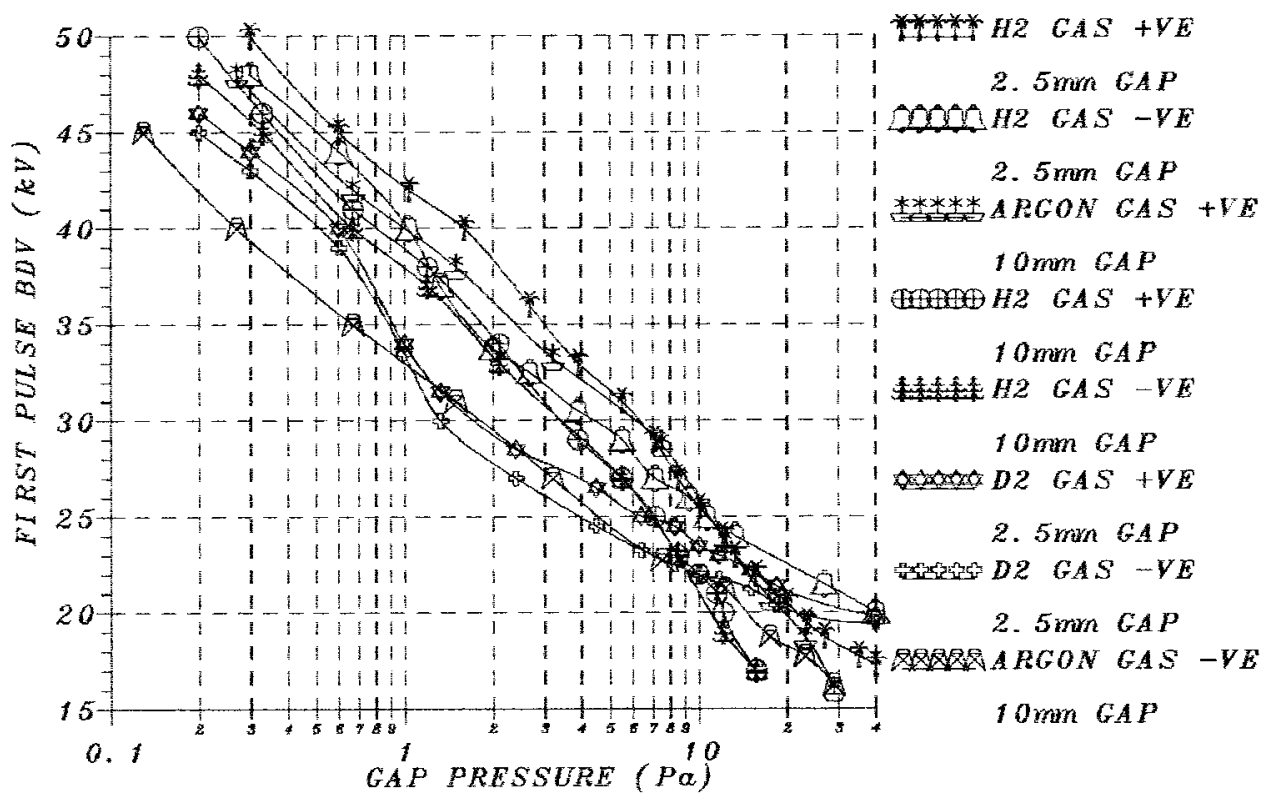

Fig. 15. First pulse breakdown voltage as a function of gap pressure characteristics. $\mathrm{Ar}, \mathrm{H}_{2}$, and $\mathrm{D}_{2}$ gases, $2.5 / 10 \mathrm{~mm}$ gaps, - ve/+ve polarities, 5.2 and $3.3 \Omega$ loads.

characteristics. The corresponding gap pressures are noted for these BDV's, from Fig. 15. From the above the lowest pressure reached during recovery process can be estimated. The gap pressures interpreted by this method is accurate close to and higher than full recovery time. This method is not accurate for lower recovery times due to reduction of BDV's from left over ionizations. The normal gap pressure as a function of lowest gap pressure during recovery characteristics are shown in Figs. 19 and 20. The full recovery pressures should be on the line of the normal gap pressure as a function of normal gap pressure, which is linear, shown in the above figures.
The experiments to measure the pressure in the gap after discharge are very complicated. However, the pressure can be measured by a) microwave interferrometer rated $100 \mathrm{GHz}$ [13] or b) laser interferrometer [14] by scanning the gap volume.

An attempt has been made to analyze the recovery times of low pressure sparkgap by a) anode temperature rise and decay similar to Frind et al. [15] and b) free and ambipolar diffusion (spherical/cylindrical) of plasmas [16]. The recovery time characteristics of argon and hydrogen gases shows good agreement for $5 \mathrm{kA}$ load current with solid phase recovery times at low pressures $(<5.5 \mathrm{~Pa})$ for - ve polarity. The 


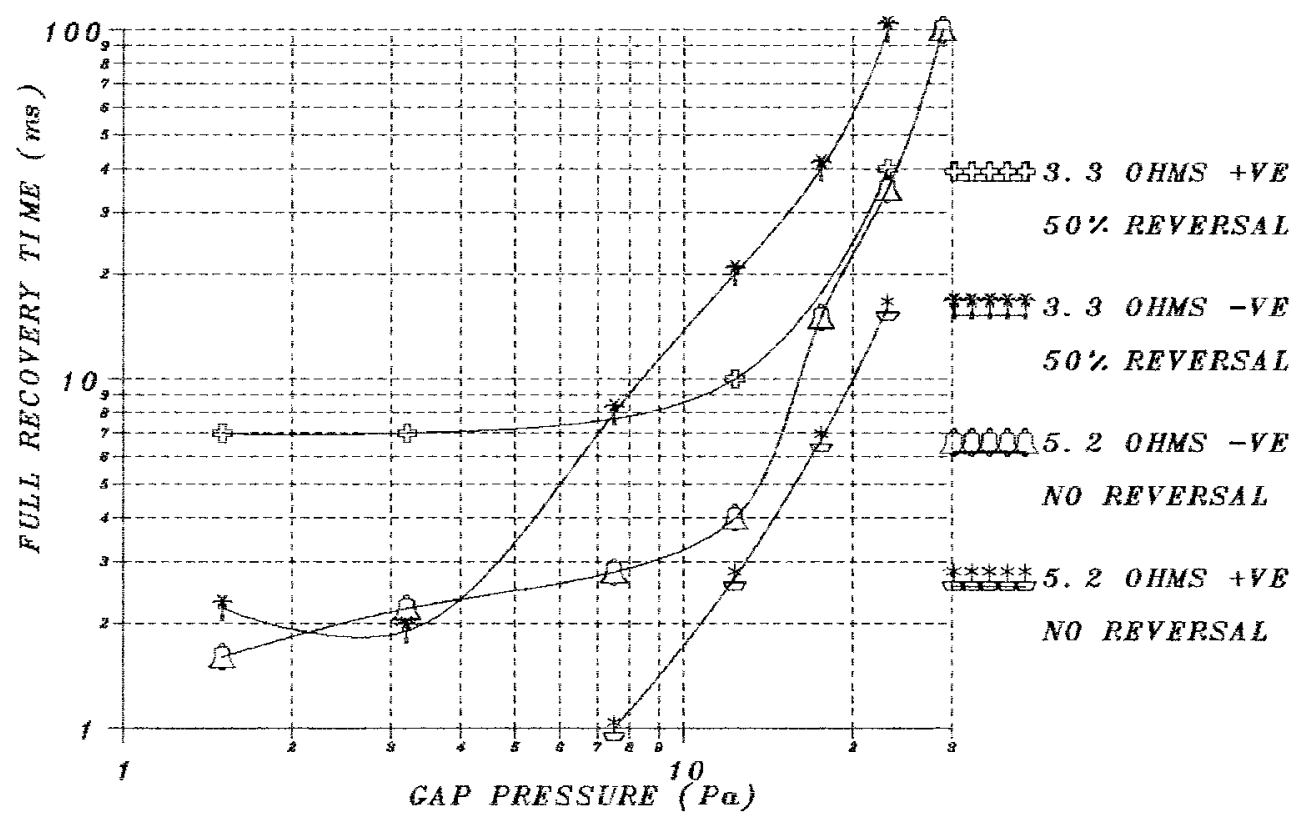

Fig. 16. Full recovery time as a function of gap pressure characteristics, argon gas, $10 \mathrm{~mm}$ gap, -ve/+ve polarities, and 5.2/3.3 $\Omega$ loads.

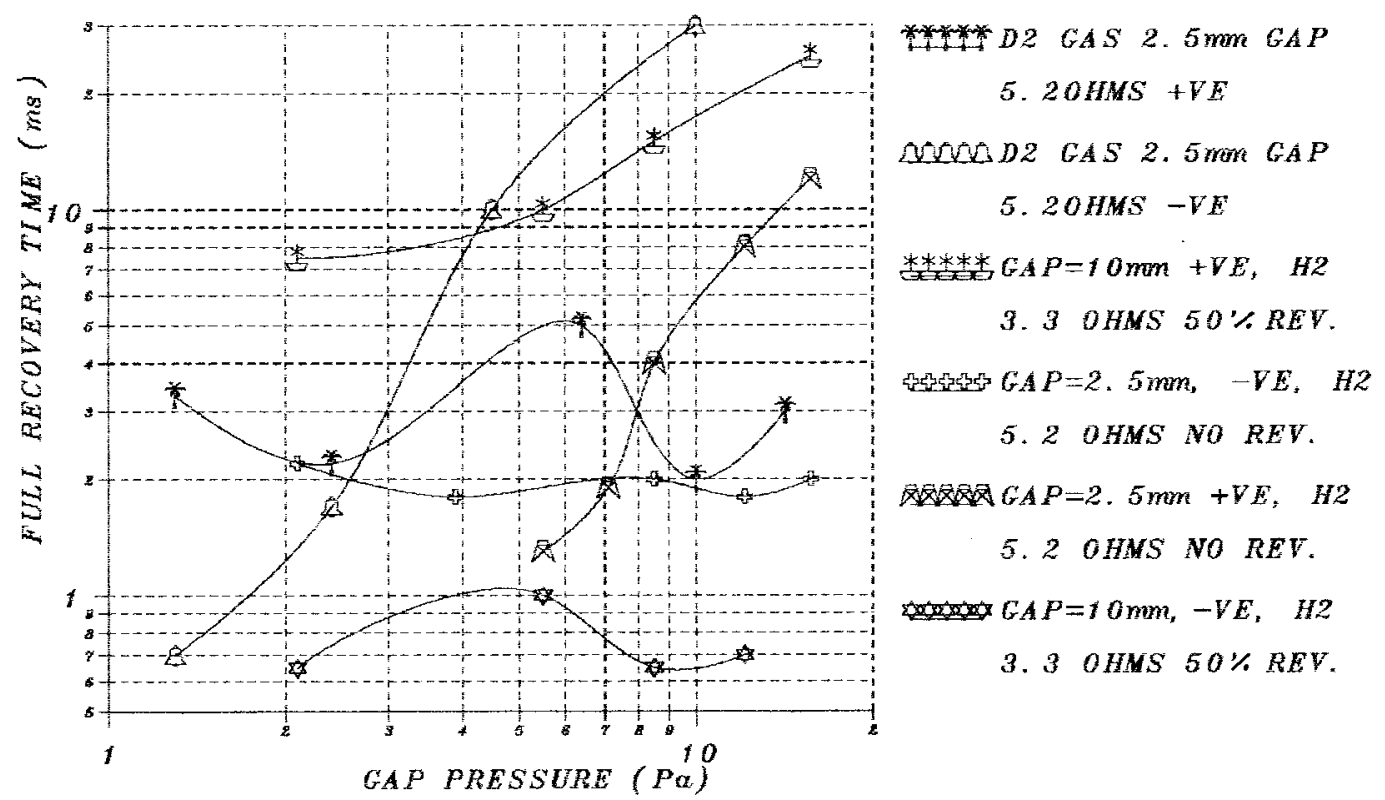

Fig. 17. Full recovery time as a function of gap pressure characteristics hydrogen and deuterium gases, $2.5 / 10 \mathrm{~mm}$ gaps, - ve/+ve polarities, and $5.2 / 3.3 \Omega$ loads.

spherical diffusion recovery times shows good agreement with experimental results at higher pressures for - ve polarity $(>5.5$ $\mathrm{Pa})$. There is a large difference in these recovery times for $+\mathrm{ve}$ polarity, due to nonuniformity in the gap configuration.

\section{Discussions}

The over recovery is predominant in case of large current reversals due to a large temperature rise in discharge and corresponding reduction in pressure. The full recovery times are approximately the same for both positive and negative polarities with no current reversals. It reduces for negative polarity and increases for positive polarity with current reversals, compared to no current reversals. This is due to edge effects of the gas inlet electrodes [6], which affects the diffusion of positive ions for different polarities and results in slower recovery for positive compared to negative polarity. It may be noted that for BDV under positive polarity, no current reversal (Figs. 6 and 12) is higher than 50\% current reversal (Figs. 8 and 10). The BDV's are 20 and $28 \mathrm{kV}$ (argon gas) at a pressure of $12.3 \mathrm{~Pa}$ with $50 \%$ and no current reversal, respectively. The lower BDV resulted in lower current in the discharge and less 


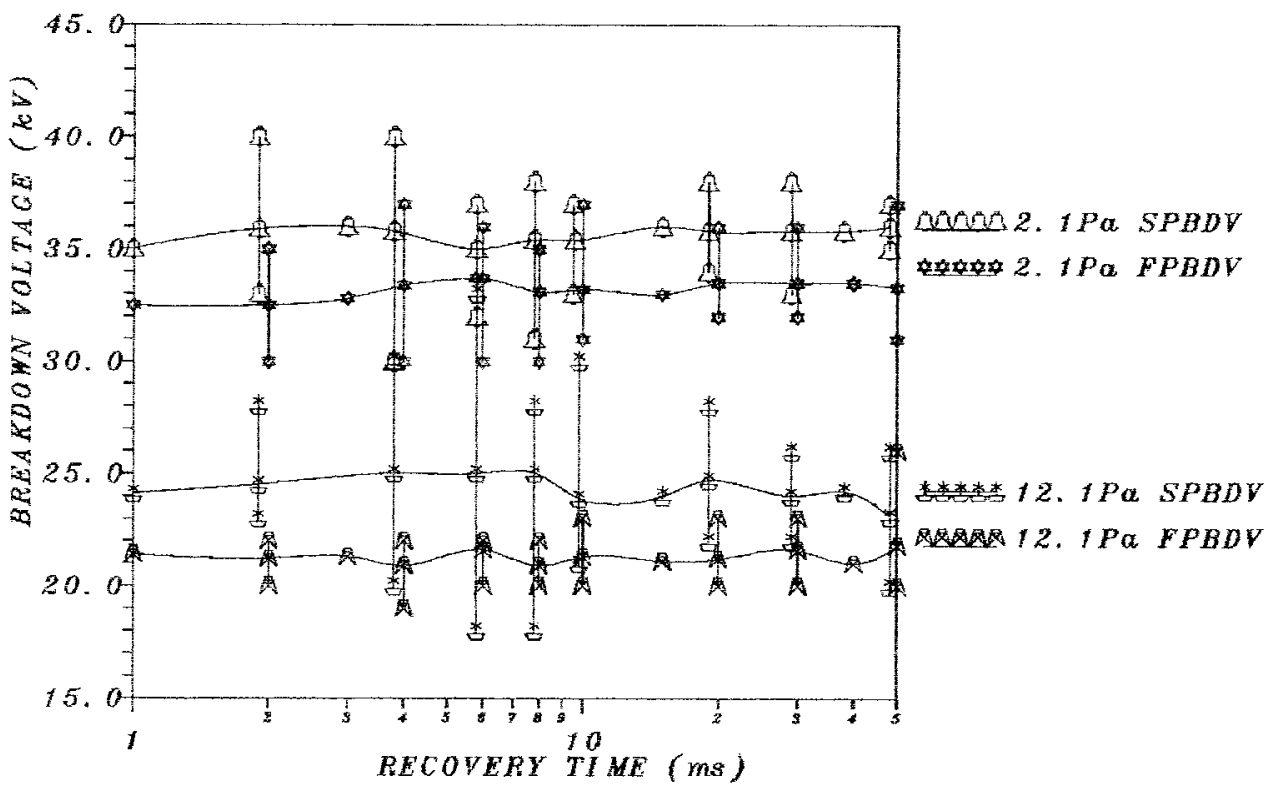

Fig. 18. Significance of over recovery with range of breakdown voltages for hydrogen gas, $10 \mathrm{~mm}$ gap, -ve polarity, $50 \%$ current reversal, $3.3 \Omega$ load. (SPBDV's are shifted toward left to avoid over lapping.)

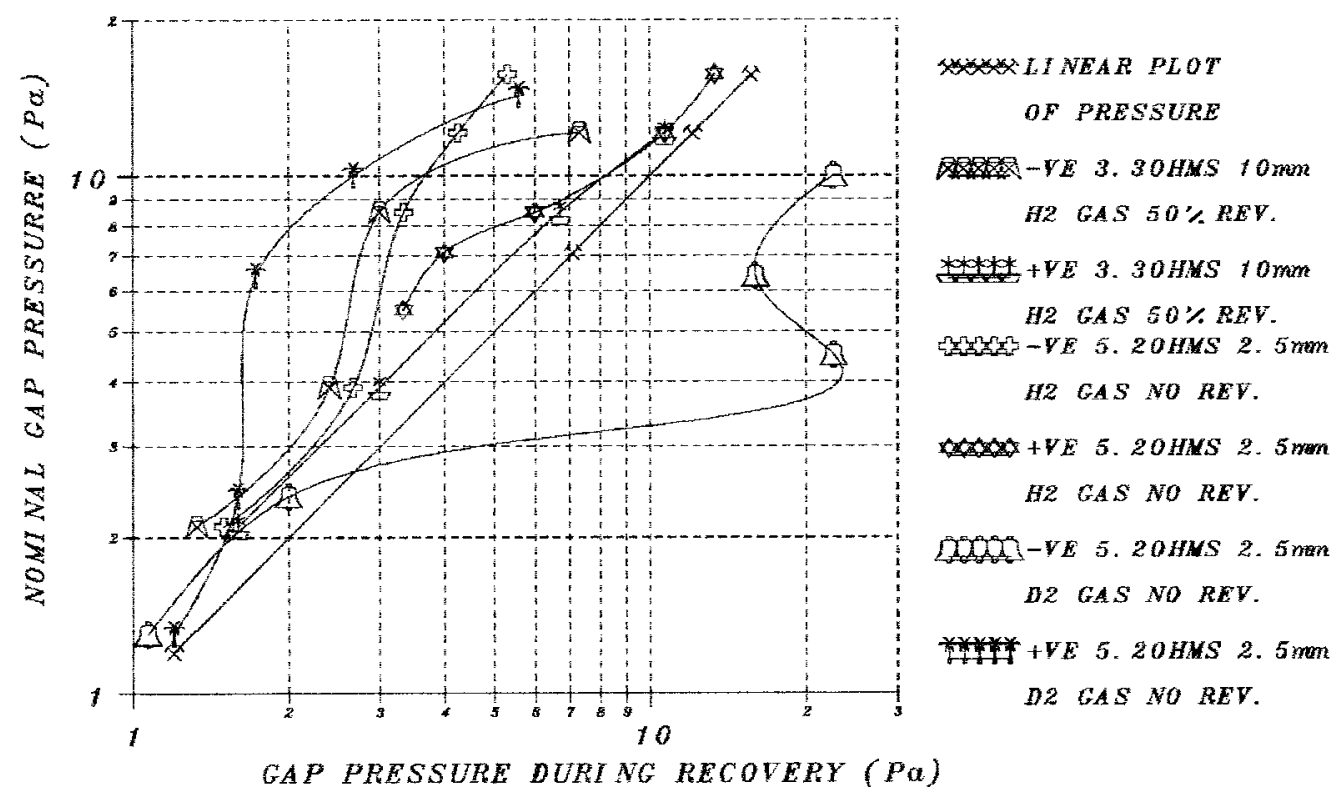

Fig. 19. Lowest gap pressure during recovery period of low pressure sparkgaps, hydrogen/deuterium gases, $2.5 / 10 \mathrm{~mm}$ gaps, +ve/-ve polarities, $5.2 / 3.3 \Omega$ loads.

reduction in gap pressure. This resulted in large recovery time for positive polarity with $50 \%$ current reversal.

The full recovery time with no current reversal is generally lower for negative polarity compared to positive polarity. However in case of $5.2 \Omega, 10 \mathrm{~mm}$ gap, argon gas, the recovery time of positive polarity is low compared to negative polarity. This is due to higher BDV with positive polarity $(32.5 \mathrm{kV})$ compared to negative polarity $(22.5 \mathrm{kV})$ at $7.5 \mathrm{~Pa}$. Whenever the BDV's and the currents are the same as that of pulsed BDV characteristics, the recovery times are very close to each other. The full recovery time for deuterium gas increases linearly with increase in pressure for negative polarity due to lower breakdown voltages compared to Fig. 15 for pressures $>4.5$ $\mathrm{Pa}$ and nearly consistent for positive polarity. However the spread in recovery time is large in case of positive polarity.

The over recovery of the short gap ( $2.5 \mathrm{~mm}$ gap) has been observed over a wide pressure range compared to long gaps (10 mm gap) for hydrogen gas. Short gaps have same full recovery times with less discharge current compared to long gaps. In short gaps, the electrons have to move only a short distance, to produce avalanche current growth. This requires a higher accelerating potential across the gap for breakdown 


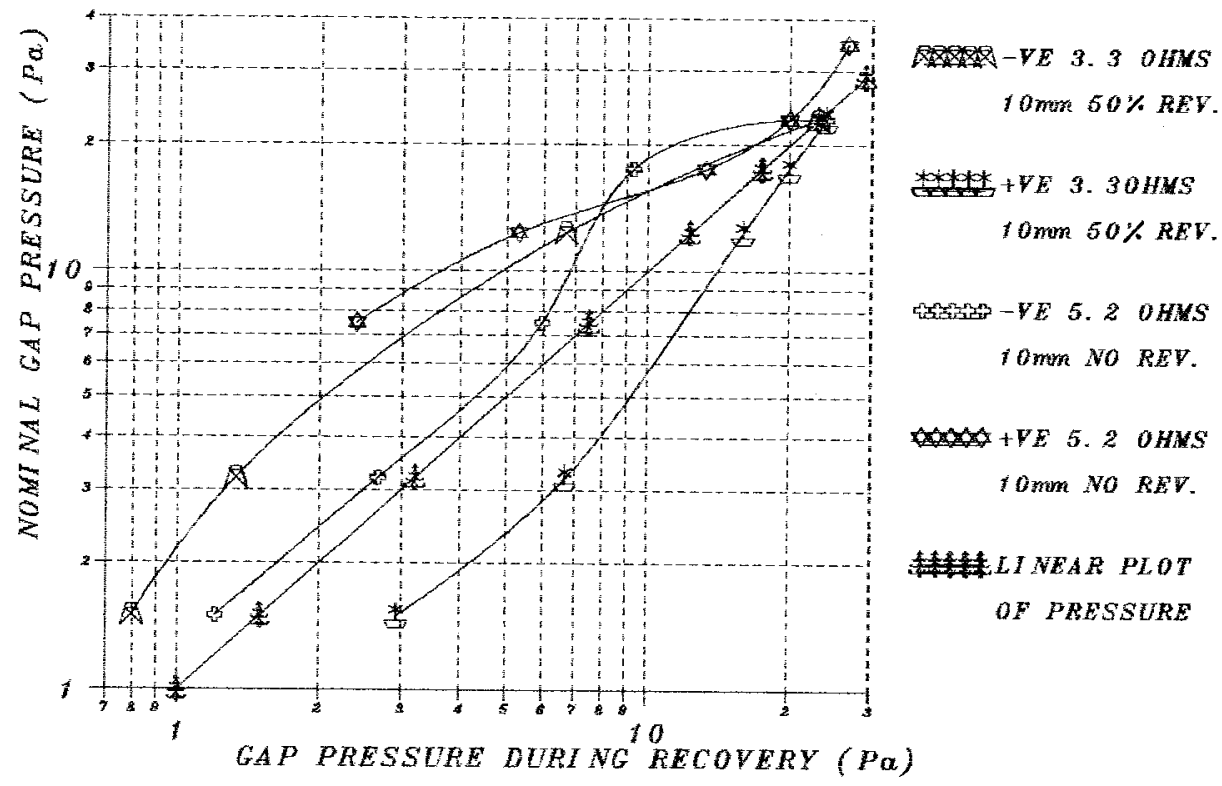

Fig. 20. Lowest gap pressure during recovery period of low pressure sparkgaps, argon gas, $10 \mathrm{~mm}$ gap, +ve/-ve polarities, $5.2 / 3.3 \Omega$ loads.

and hence higher current in the gap. This results in larger reduction in pressure in the gap. The BDV's at a pressure of $12.1 \mathrm{~Pa}, 10 \mathrm{~mm}$ gap, (hydrogen gas, Figs. 9 and 10) are 21.5 and $20 \mathrm{kV}$ with $50 \%$ current reversal, against 27 and $22.5 \mathrm{kV}$ for $2.5 \mathrm{~mm}$ gap with no current reversal (Figs. 11 and 12) for negative and positive polarities, respectively. This means that when the breakdown takes place with electrons' collisions in the gap having mean free path less than gap spacing, the recovery of the gap is slow. The probability of over recovery is very low. When the breakdown takes place with field initiated electron avalanche in the gap having mean free path greater than gap spacing, the recovery is fast. The probability of over recovery is very high. The recovery is slow with increase in pressure in case of positive polarity with no current reversal. However recovery time is less when compared to positive polarity with $50 \%$ current reversal. The lowest pressures reached during discharge are lower than the normal operating pressures, for the cases with over recovery of the gap (Figs. 19 and 20). Generally if the lowest pressures are above the nominal pressure characteristics, it will result in over recovery of the gap. It will result in under recovery if the lowest pressures are below this characteristics for that delay time or less. However there can be some exceptions. Over recovery has been observed for gap pressure of $4.5 \mathrm{~Pa}$, negative polarity, due to very low BDV $(18 \mathrm{kV})$ compared to BDV from Fig. 15 (25 kV) [12]. An order of magnitude faster recovery can result in a gap with $50 \%$ reduction in $\mathrm{BDV}$. This lower BDV at $4.5 \mathrm{~Pa}$ has resulted in a small over recovery with a large recovery time compared to 1.3 and 2.4 Pa gap pressures. It is evident from the above that the pressure during sparkgap recovery process should reduce lower than the nominal gap pressure for over recovery of the gap.

\section{CONCLUSIONS}

The over recovery of low pressure sparkgaps can be observed along the left-hand side of Paschen's minimum. It depends upon parameters like pressure, gas species, current, and current reversal. Conditioning of sparkgap before the recovery experiments in addition to initial conditioning, results in consistent BDV's and helps in over recovery with reduced recovery times in case of low pressure sparkgaps. The over recovery can been observed up to certain gap pressures and only full recovery for higher pressures. Generally full recovery times increase with increase in pressures for positive polarity and are nearly consistent with increase in pressure for -ve polarity. The sparkgap recovery is faster under negative polarity compared to positive polarity in case of low pressure sparkgaps having some nonuniformity. The recovery is faster with low molecular weight gas like hydrogen and deuterium gases compared to argon gas. The hydrogen gas, negative polarity has the fastest recovery characteristics followed by deuterium and argon. Short gap recovers faster than long gaps. The pressure during recovery of the gap reduces below nominal value in case of over recovery. The reduction in pressure due to first pulse discharge is higher at low pressures and lower at high pressures.

\section{ACKNOWLEDGMENT}

The authors are thankful to staff members at Accelerator and Pulse Power Division for useful discussions and support. They would also like to thank Dr. S. S. Kapoor, Director, Physics and E\&I Groups, BARC, for his keen interest and encouragement.

\section{REFERENCES}

[1] K. V. Nagesh, "A study of recovery times of low pressure sparkgaps," $\mathrm{Ph} . \mathrm{D}$. dissertation, Indian Institute of Science, Bangalore, July 1997.

[2] G. A. Mesyats and D. I. Proskurovsky, Pulsed Electrical Discharges in Vacuum. New York: Springer-Verlag, July 1988, ch. 4.53.

[3] K. Tsuruta and H. Ebara, "Modeling of a gas temperature decay after arc discharge in small air gaps," IEEE Trans. Elect. Insulation, vol. 27, pp. 451-456, June 1992. 
[4] K. Tsuruta, I. Takahashi, T. Ojima, and Y. Komuro, "Experimental study of the voltage recovery characteristics of small air gaps," IEEE Trans. Plasma Sci., vol. 17, pp. 560-564, June 1989.

[5] E. J. Lauer and D. L. Brix, "Breakdown and recovery of a $250 \mathrm{kV}$ low pressure sparkgap," IEEE Trans. Plasma Sci., vol. PS-10, pp. 294-297, Oct. 1982.

[6] P. Osmokrovic, I. Krivokapic, and Kristic, "Mechanism of electrical breakdown left of paschen's minimum," IEEE Trans. Dielect. Electr. Insulation, vol. 1, no. 1, pp. 77-81, Feb. 1994.

[7] W. Nelson, "Analysis of the accelerated life test data-Part-I, The Arrhenius model and graphical methods," IEEE Trans. Elect. Insulation, vol. EI-26, pp. 165-181, Dec. 1971.

[8] IEEE Guide for the Statistical Analysis of Thermal Life Test Data, IEEE Standard 101, 1972.

[9] H. Hirose, "Maximum likelihood estimation in the three-parameter Weibull distribution," IEEE Trans. Dielect. Electr. Insulation, vol. 3, pp. 43-55, Feb. 1996.

[10] M. Cacciari, G. Mazzanti, and G. C. Montanari, "Weibull statistics in short term dielectric breakdown of thin polyethylene films," IEEE Trans. Dielect. Electr. Insulation, vol. 1, pp. 153-159, Feb. 1994.

[11] C. Laurent, C. Chauvet, and J. Berdala, "The significance of the Weibull threshold in short term breakdown statistics," IEEE Trans. Dielect. Electr. Insulation, vol. 1, no. 1, pp. 160-162, Feb. 1994.

[12] S. L. Moran, M. G. Grothaus, and L. W. Hardsty, "Hydrogen spark switches for REP-rated accelerators," Proc. Int. Conf. High Power Particle Beams, Part III, Washington, DC, May 25-29, 1992, p. 726.

[13] H. Hermansdorfer, "Microwave Diagnostics Techniques" in Plasma Diagnostics, W. Lochte-Holtgreven. Ed. Amsterdam, The Netherlands: North Holland, 1968, ch. 8, p. 478.

[14] S. M. Turnbull, S. T. Macgregor, F. A. Tuema, and O. Farish, "A quantitative laser Schliren method for measurement of neutral gas density in high pressure gas switches," Meas. Sci. Technol., vol. 4, pp. $1154-1159,1993$.

[15] G. Frind, J. J. Carrol, and E. J. Tuohy, "Recovery times of vacuum interruptors which have stationary anode spots," IEEE Trans. Power App. Syst., vol. 101, pp. 775-781, Apr. 1982.

[16] Y. P. Raizer, Gas Discharge Physics. New York: Springer-Verlag, 1991, pp. 69, 134.

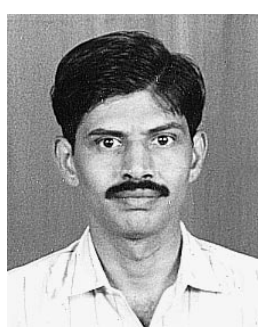

K. V. Nagesh received the B.E. degree in electrical power in 1972 and the M.E. degree in power system engineering in 1974 from University of Mysore. He received the Ph.D. degree in 1998 from High Voltage Engineering Department, Indian Institute of Science, Bangalore, India.

He joined Bhabha Atomic Research Center, Mumbai, India, in 1975. He is associated with the development of pulse power systems based on Marx generators and Tesla transformers, capacitor banks, pulsed magnetic field coils for remote repositioning in power reactor applications, and induction electron accelerators based on magnetic pulse compression.
P. H. Ron received the B.E. degree from Poona University in 1961, the M.Sc. (Eng.) degree from the University of Manchester Institute of Science and Technology in 1969, and the Ph.D. degree from the Indian Institute of Science, Bangalore, in 1984.

He was a visiting Scientist at the National Research Council, Ottawa, Canada, in 1985 and at McGill University, Montreal, Canada, in 1987. $\mathrm{He}$ has been working in the fields of high voltage insulation engineering, multigigawatt pulse power systems, pulsed high magnetic fields, EMI/EMC, and industrial electron accelerators.

G. R. Nagabhushana, photograph and biography not available at the time of publication.

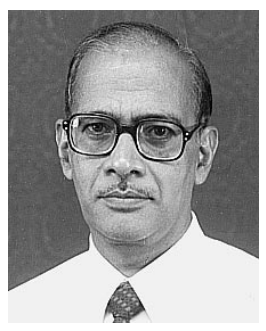

R. S. Nema received the Ph.D. degree from Saugar University in 1968 .

He joined the High Voltage Engineering Department, Indian Institute of Science, Bangalore, India, in 1968. His areas of interest and work are in dielectrics and insulation, insulation diagnostics, and partial discharges. His group has been instrumental in developing a phase-related pulse-height analyzer for signature pattern analysis of partial discharge distributions related to defects in insulation. He has published more than 100 papers including a number of papers at the IEEE on $\mathrm{SF}_{6}$ insulation, partial discharge measurement on oil-press board transformer insulation, and polypropylene insulation used in capacitors.

Dr. Nema is a member of National Sub-Committee of CIGRE on insulation materials. He is also a convener of EHV Technology Conferences in India. 